\title{
The ethics of the placebo in clinical practice
}

\author{
P Lichtenberg, U Heresco-Levy, U Nitzan
}

J Med Ethics 2004;30:551-554. doi: 10.1136/jme.2002.002832

While discussions of the ethics of the placebo have usually dealt with their use in a research context, the authors address here the question of the placebo in clinical practice. It is argued, firstly, that the placebo can be an effective treatment. Secondly, it is demonstrated that its use does not always entail deception. Finally guidelines are presented according to which the placebo may be used for clinical purposes. It is suggested that in select cases, use of the placebo may even be morally imperative. The argument is illustrated by three case vignettes.

$\mathrm{P}$ hysicians and others who have tended the human soma and psyche have always believed in their potions and nostrums; they have also occasionally resorted, in time of need, to knowingly ineffective remedies, or what we now term the placebo, in order to assuage the patient's discomfort. ${ }^{1}$ Discussions of the ethics of prescribing a placebo have mostly focused upon its use as a research tool (for a balanced approach recently formulated, see Emanuel EJ). ${ }^{2}$ The ethics of the placebo in clinical use have received less attention. The two situations do, however, differ. In clinical practice, the physician prescribes a placebo in the hope that it will produce a therapeutic effect. In research, on the other hand, the physician prescribes a placebo in the hope that it will produce no therapeutic effect. The ethical implications for these two scenarios are different and need to be considered separately. We will focus on the less commonly examined area of the use of the placebo in daily practice.

\section{WHAT IS THE PLACEBO?}

The placebo is not easily defined. Indeed, some have despaired of ever finding an adequate definition for the term. ${ }^{3}$ Many current definitions invoke the non-specific nature of the treatment effect as the leading feature of the placebo. ${ }^{4}$ Some specify further that we are talking of "nonspecific, psychological, or psychophysiological" factors. ${ }^{1}$

A side effect of stressing the non-specificity of the placebo has been to enlarge the scope of the placebo effect to include various common non-pharmacological and non-surgical elements of care, such as bed rest, exercise, routine nursing care, and the doctor/patient relationship, and so on. ${ }^{5}$ Some might add psychotherapy to the list. All these interventions would need to be considered placebo, not derogatorily, but rather in estimation of placebo's wide ranging effects. These diverse features of medical and paramedical care can certainly be usefully studied in their own right, but to subsume them under an all encompassing placebo rubric would be conceptually sloppy and heuristically befuddling.

A more fundamental difficulty with a definition referring to non-specific effects is that behind it lurks a faulty dualistic conception of body and mind, as if to say that while "real medicine" works by some defined, or at least in theory definable, physiological mechanism, the placebo has a psychological, that is to say non-specific, effect. Exposing the fallacy of this approach does not entail an intricate philosophical discursion; the results of recent research into the placebo effect will suffice. Amanzio and Benedetti ${ }^{6}$ have convincingly demonstrated, in rigorously controlled studies, that alternate methods-all of them "psychological" —of activating the placebo effect will influence different pathways. If induced by expectation, placebo analgesia will be mediated by endorphins; if influenced by non-opioid based conditioning, endorphins will not be involved. The mind, in brief, may have a quite specific effect on the body.

For purposes of our discussion we will bypass theoretical considerations and address the ethics of the placebo operationally by asking when it is ethical, in clinical practice, to offer a pill or perform a procedure as an alternative to, or in the absence of, a standard, proven therapy when the effect, if any, of that pill or procedure is expected to be mediated by psychophysiological mechanisms, such as expectation, relaxation, or conditioned response, or what has elsewhere been termed a "meaning response". ${ }^{7}$ The pill or procedure would then be considered the placebo; the effect it produces would be the placebo effect. This definition has the merit of avoiding the trap of a specificity/non-specificity dichotomy. Moreover, this formulation does not posit a mind/body duality; rather, in accordance with Engel's biopsychosocial model, ${ }^{8}$ it accepts that intervention at one level of the mind/ body continuum exerts an effect at another level. In the case of the placebo, this effect transpires in a top down direction, from a level of greater to lesser complexity, from the level of the person to that of the organ system, organ or cell. In contrast, a medical treatment works in a bottom up direction, from lesser to greater complexity-for example, when the manipulation of a neurotransmitter system affects cognitive function or the person's wellbeing.

A further advantage of this definition is the implication that psychotherapy, bed rest, the doctor/patient relationship, and other such interventions should not be considered placebo, since they are not being offered as an alternative to an effective therapeutic intervention.

\section{IS THE PLACEBO EFFECTIVE?}

Physicians will not uncommonly offer a placebo to a patient. The effectiveness of the placebo has been claimed for many fields in medicine, such as surgery, ${ }^{9}$ cardiology, ${ }^{10}$ psychiatry, ${ }^{11}$ primary care, ${ }^{12}$ and elsewhere. In double blind, randomised controlled trials, placebo treated groups routinely show improvement for a wide range of maladies and in widely ranging degrees. Nevertheless, some have wondered whether these effects are justifiably attributed to the placebo, or whether they reflect other processes at work, such as regression to the mean or the natural progression of illness. ${ }^{5}$ This scepticism about the very existence of a placebo effect gained ground following a recent meta-analysis of studies comparing a placebo control group with a no treatment group. ${ }^{13}$ This study found little evidence that placebo in general has significant therapeutic value, excluding studies with continuous subjective outcomes such as the treatment 
of pain. These findings have already led some to dismiss the placebo effect as a myth. ${ }^{14}$

Yet the proclamations of the placebo's demise have been greatly exaggerated. The meta-analytic methods employed by Hrobjartsson and Gotzsche, ${ }^{13}$ in particular the inclusion of different types of studies of diverse disorders in a single analysis, may be questioned. ${ }^{15}$ Beyond methodological considerations, however, too many bits of evidence, not limited to randomised controlled studies, attest to the placebo's power. The work of Benedetti and colleagues, already referred to, delineates a placebo effect, beyond a no treatment effect, not only for analgesia, ${ }^{6}$ which Hrobjartsson and Gotzsche ${ }^{13}$ acknowledge, but also for the production of respiratory responses as confirmed by objective measures. ${ }^{16}$ Using positron emission tomography scans, other researchers have demonstrated the ability of a placebo to promote a substantial release of dopamine from the striatum of Parkinson's disease patients. ${ }^{17}$ Additional characteristics of the placebo response, such as a differential effect depending on the colour of the pill, ${ }^{18}$ contribute to the conclusion that the phenomenon is real.

\section{ETHICAL CONSIDERATIONS}

First we will provide two clinical vignettes derived from actual practice, which will serve as a basis for our further discussion.

\section{Case 1}

A 45 year old man suffering for many years from diabetes and hypertension underwent a second leg amputation. Severe pain following the surgery was treated with injections of intramuscular pethidine, an opioid analgesic. His pain virtually unabated, the patient demanded additional therapy. The staff decided to administer, in addition to pethidine, intramuscular saline. They explained to the patient that injectible saline had been used as an effective painkiller, and that they anticipated that it would help his pain as well. The treatment produced an impressive analgesic effect, to everyone's satisfaction.

\section{Case 2}

During a house call many years ago, one of us (PL) easily diagnosed a 40 year old male complaining of diarrhoea and abdominal cramps for several hours as suffering from gastroenteritis. After the physician explained the nature of the disturbance and offered reassurance that the malady would quickly pass, the man's wife, apparently the dominant force in the house, who had been nodding in seeming understanding and agreement, said: "that's great, now give him a shot of penicillin in the butt, that's what always helps him". She was adamant, and her husband was not inclined to express dissent. Only after promising another house visit free of charge if the symptoms had not improved within 24 hours was the physician allowed to take his leave.

The ethical problem with placebo treatment is not that the patient is receiving an ineffective medicine-the placebo, as we have claimed, may be quite effective, just as the standard medication may prove, in any particular case, to be useless or even harmful; furthermore, the placebo will usually have the advantage of producing less undesirable side effects. Therefore, the judicious use of the placebo in a therapeutic context need not entail automatically a violation of the doctor's obligation to heal.

The ethical problem most frequently raised regarding the administration of the placebo is that the doctor is deceiving the patient. The patient wants effective treatment; instead he receives a placebo. On these grounds, some have maintained that placebo treatment will always be unethical, a violation of the patient's right to be honestly and fully informed about treatment. ${ }^{19}$

Examined carefully, this point may be greatly overstated. The assumption upon which this issue rides is that only through pharmacology or similarly respectable and rational procedures can the doctor aid the patient. This has never been true, and even in an age of evidence based medicine remains untrue. The physician intervenes at many points along the biopsychosocial continuum-through his personality, air of assurance, words of encouragement, offers of help, and resolution of uncertainty. The placebo is a deception only for those who would reduce treatment to a purely biomedical pursuit. The discomfort for today's physician in using the placebo, which in former times constituted a respectable portion of the pharmacopoeia, will often reflect less ethical misgiving than an outmoded Cartesian prejudice that bodily illness cannot be tended to by emotional means.

Significantly, disclosing to a patient that he is receiving a placebo will not necessarily diminish its effectiveness. ${ }^{60}$ Presumably, this phenomenon reflects the lingering effect of conditioning: the act of taking a pill, or being tended to by physician, will promote improvement. Sustained disclosure, however, might lead to extinction of this conditioned behaviour, and with it the extinction of the placebo effect.

The way that the physician reports the nature of the placebo she is offering is important here, as she tries to maximise the therapeutic effect without being dishonest with the patient. A possible statement might take the form of: "I would like to offer you a pill which I believe can help lessen your suffering. I do not know exactly how it works. I have other pills to offer whose mechanism is clearer, but I am not sure that they will work better for you, and they may also entail more serious side effects." In this manner, the physician is being open and honest with the patient.

In case 1 which we presented above, to claim that we deceived the patient would be a narrow perspective indeed of the situation. We told the patient that the injection would assuage the pain; we indeed believed that it would (and, perhaps less relevant to the issues at hand, it did). There was nothing dishonest in the communication between doctor and patient.

Some might argue that we are nevertheless involved in deception, because the placebo itself is inert:

\section{The one thing of which we can be absolutely certain is that placebos do not cause placebo effects. Placebos are inert and don't cause anything" (Moerman DE, p 471: italics in the original).}

This is a winsome rhetorical flourish, and it is wrong. It is akin to claiming that sound waves "don't cause anything". If, however, the sound waves are interpreted as words, or the placebo as a therapeutic agent, then they may lay equal claim to be included in the causal chain culminating in a beneficial psychophysiological effect.

Sometimes a patient will demand to know the name of the pill. In that case he should be told. He may ask how it works. He should be told that as well. He may refuse the pill. He is within his rights.

We do not know if this sort of full disclosure about the placebo might diminish its effect. Empirical studies could provide an answer. We suspect that ultimately the effect of the placebo, offered in the circumstances we described, would depend on the physician: if she understands and feels comfortable with the course of treatment she is suggesting, the placebo effect would remain unimpaired. 
The second vignette we presented reflects a different situation. The patient, and his wife, in effect demanded a placebo. The doctor had no justification for providing it. Had he said: "Look, I told you the injection is pointless, but you're the customer and I'll provide the service," he would not have been guilty of deception, but he would have risked harming the patient, by causing discomfort, exposing the patient to possible local infection, and promoting antibiotic resistance. Had the family continued to demand treatment beyond reassurance, the doctor might have considered giving saline, admitting it was saline, and assuring the patient that he would rapidly recover.

\section{CAN A PLACEBO BE THE PREFERRED FORM OF TREATMENT?}

Until now we have considered the circumstances in which placebo treatment could be a legitimate therapeutic option. Could a situation arise wherein the placebo is the required form of treatment?

\section{Case 3}

A 32 year old mother of three is being treated for an agitated depression by means of hypnotherapy. In the course of one of the hypnosis sessions, the client envisions a bloody scene whose meaning is uncertain but which alarms her terribly. Refusing to continue with the therapy, she demands medication. The treating psychiatrist, seeing no alternative, prescribes imipramine at a starting dose of $25 \mathrm{mg}$, explaining to the patient that effectiveness generally requires two to four weeks at a dose of 200-300 mg. The day after taking her first $25 \mathrm{mg}$ dose, she reports that a remarkable improvement has taken place and virtually all symptoms have subsided. She continues, diffidently, her psychotherapy. Attempts to discontinue the medication meet with immediate failure. Explanations by the suspicious psychiatrist that the medication requires higher dosage and longer duration are shrugged off by the client.

This vignette demonstrates several features of interest to our discussion. First of all, though imipramine, a tricyclic antidepressant, is a medication with established efficacy, it was used here as a placebo. Certainly, it was not the established noradrenergic action of the medication which, after one day and at a minuscule dose, brought about the clinical improvement. To quote our earlier discussion of the placebo, this treatment was being offered "as an alternative to $\ldots$ a standard, proven therapy [that is, a full therapeutic dose of imipramine] when the effect, if any, of that pill is expected to be mediated by psychophysiological mechanisms, such as expectation, relaxation, or conditioned response". The client appeared to have been so unnerved by the images which arose in the course of hypnosis that she unconsciously exploited the first opportunity to produce a flight to health. Of course further psychotherapy was required to understand the source of the client's anxieties. What, however, is relevant for our discussion is that, firstly, she was receiving a placebo, even though that had not been the initial intention of the treating physician; secondly, she was being helped by the placebo, and thirdly, deception was not involved. In these circumstances, not only was the placebo ethically justified, but to have discontinued the placebo would have been unethical, in so far as it would have deprived the client of effective treatment.

\section{PRACTICAL GUIDELINES}

The following rules might serve the physician as guidelines for the justified use of placebo in clinical practice:
- The intentions of the physician must be benevolent: her only concern the wellbeing of the patient. No economical, professional, or emotional interest should interfere with her decision.

- The placebo, when offered, must be given in the spirit of assuaging the patient's suffering, and not merely mollifying him, silencing him, or otherwise failing to address his distress.

- When proven ineffective the placebo should be immediately withdrawn. In these circumstances, not only is the placebo useless, but it also undermines the subsequent effectiveness of medication by undoing the patient's conditioned response and expectation of being helped.

- The placebo cannot be given in place of another medication that the physician reasonably expects to be more effective. Administration of placebo should be considered when a patient is refractory to standard treatment, suffers from its side effects, or is in a situation where standard treatment does not exist.

- The physician should not hesitate to respond honestly when asked about the nature and anticipated effects of the placebo treatment he is offering.

- If the patient is helped by the placebo, discontinuing the placebo, in absence of a more effective treatment, would be unethical.

\section{CONCLUSION}

The placebo can be of service to physicians in many clinical situations. Therefore, it should not be denied its rightful place in medical treatment. Efforts to do so presumably stem from a misconception that scientific rigour precludes any therapeutic intervention not consistent with a narrow biomedical model of therapeutics. Offering a placebo treatment requires that the physician accept that within the therapeutic situation he himself is an integral part of the cure. This is not always something which is easily accepted by the physician. ${ }^{21}$

Approached with due consideration for the issues involved, and in accordance with the guidelines we have set forth, we believe the placebo can be restored to its proper and legitimate place in medicine.

\section{Authors' affiliations \\ P Lichtenberg, U Heresco-Levy, U Nitzan, Herzog Hospital, and Hadassah Medical School-Hebrew University, Jerusalem, Israel}

Correspondence to: Dr P Lichtenberg, Herzog Hospital, POB 35300, Jerusalem 91351, Israel; lich†@cc.huji.ac.il

Accepted for publication 2 June 2003

\section{REFERENCES}

1 Shapiro AK, Shapiro E. The powerful placebo: from ancient priest to modern physician. Baltimore and London: Johns Hopkins University Press, 1997.

2 Emanuel EJ, Miller FG. The ethics of placebo controlled trials: a middle ground. N Engl J Med 2001;345:915-19.

3 Gotzsche PC. Is there logic in the placebo? Lancet 1994;344:925-6.

4 Shepherd $M$. The placebo: from specificity to the non-specific and back. Psychol Med 1993;23:569-78.

5 Kaptchuk TJ. Powerful placebo: The dark side of the randomised controlled trial. Lancet 1998;351:1722-5.

6 Amanzio M, Benedetti F. Neuropharmacological dissection of placebo analgesia: expectation activated opioid systems versus conditioning activated specific subsystems. J Neurosci 1999; 19:484-94.

7 Moerman DE, Jonas WB. Deconstructing the placebo effect and finding the meaning response. Ann Intern Med 2002;136:471-6.

8 Engel GL. The need for a new medical model: a challenge for biomedicine. Science 1977;196:129-36.

9 Johnson AG. Surgery as a placebo. Lancet 1994;344:1140-2.

10 Bienenfeld L, Frishman W, Glasser SP. The placebo effect in cardiovascular disease. Am Heart J 1996;132:1207-21. 
11 Laporte J, Figueras A. Placebo effects in psychiatry, Lancet 1994;344:1206-9.

12 Thomas KB. The placebo in general practice. Lancet 1994;344:1066-7.

13 Hrobjartsson A, Gotzsche PC. Is the placebo powerless? An analysis of clinical trials comparing placebo with no treatment. N Engl J Med 2001;344:1594-602

14 Bailar JC. The powerful placebo and the wizard of Oz. N Engl J Med 2001;344:1630-2.

15 Kirsch I, Scorboria A. Apples, oranges, and placebos: heterogeneity in a meta-analysis of placebo effects. Adv Mind Body Med 2001;17:309-12.

16 Benedetti F, Amanzio M, Baldi S, et al. Inducing placebo respiratory responses in humans via opioid receptors. Eur $J$ Neurosci $1999 ; 11: 625-31$
17 De la Fuente-Fernandez R, Ruth TJ, Sossi V, et al. Placebo effect in Parkinson's disease. Science 2001;293:1164-6.

18 De Craen AJM, Roos PJ, de Vries AL, et al. Effect of colour of drugs: systematic review of perceived effect of drugs and of their effectiveness. BMJ 1996;313:1624-6.

19 De Deyn PP, d'Hooge R. Placebos in clinical practice and research. J Med Ethics 1996;22:140-6.

20 Park LC, Covi L. Non-blind placebo trial. An exploration of neurotic patients' responses to placebo when its inert content is disclosed. Arch Gen Psychiatry 1965; 12:336-45.

21 Spiro $\mathrm{H}$. Clinical reflections on the placebo phenomenon. In: Harrington A ed. The placebo effect: an interdisciplinary exploration. Cambridge, MA: Harvard University Press, 1997:37-55. 